\title{
Queratocono: una revisión y posible situación epidemiológica en Colombia.
}

\section{Matilde Mora Valencia1, Claudia Bonilla Estévez1, Oswaldo Vargas Garzón1, Olga Lucía Giraldo Valencia ${ }^{1}$}

\author{
1. Universidad Antonio Nariño, Facultad de Optometría, Grupo de Investigación, Línea Queratocono
} Bogotá, Colombia

Correspondencia: decano.optometria@uan.edu.co

Recibido: 22-08-07 / Aceptado: 14-12-07

\section{Resumen}

En Colombia y en el mundo entero sabemos cómo definir el queratocono, lo diagnosticamos con una excelente propiedad, incluso muchas veces, aún sin ayuda de la topografía corneal, con una prueba de queratometría, los profesionales de la salud visual y ocular podemos observar miras no nítidas inductoras de queratocono. Es una degeneración corneal de causa idiopática. Decir que su origen es hereditario, puede ser tan falso como decir que su origen es metabólico. ¿Que sabemos a ciencia cierta? mucho y nada, pero la única realidad es que mientras en otros países su incidencia y prevalencia es conocida, en Colombia no tenemos datos registrados oficialmente. El registro es subclínico, pues aunque existe entre la clasificación internacional de las enfermedades, versión 10, como patología clínica, los profesionales de la salud visual y ocular normalmente no lo registran. El objetivo de este artículo es presentar una revisión general de esta patología.

Palabras claves: blefaritis, córnea, hipermetropía, queratocono, taquimetría, topografía corneal,

\begin{abstract}
In Colombia and throughout the world we know how to define keratoconus. We diagnose it with an excellent precision, usually without the aid of the corneal topography. With a test of queratometría, the professionals of the visual and ocular health can observe inductive nonclear sights of keratoconus that is a corneal degeneration of idiopatic cause. To say that its origin is hereditary, can be as false as to say that its origin is metabolic. What do we know for sure? Much and nothing at all. The only truth is that while in other countries its incidence and prevalence is well-known, in Colombia we do not have registered data officially. The registry is subclinical, because although it exists between the international classifications of the diseases, version 10, as clinical pathology, the professionals of the visual health and ocular normally do not register it. The objective of this article is to present a general revision of this pathology.
\end{abstract}

Key words: blepharitis, cornea, keratoconus, metabolic disorder, corneal topography, tachymetry. 


\section{Definición}

El queratocono es una enfermedad de la córnea, en la que ésta presenta un adelgazamiento y protruye ocasionando cambios severos en la capacidad visual del paciente. El grado de protrusión puede variar de manera importante desde una situación en la que la córnea adquiere la forma típica de cono, hasta casos sólo detectables por procedimientos clínicos especializados. Este grupo de investigación denominará queratocono en forma genérica a cualquier protrusión corneal ya sea cónica al centro, elevación en la periferia o globosa total siguiendo los planteamientos de la escuela norteamericana y los resultados de la optómetra investigadora colombiana Lucrecia Polanco de Dilences Ltda, «Verdadera forma de la córnea, sus irregularidades y su corrección», proyecto aprobado y apoyado por Colciencias. Según diferentes estudios, el queratocono es una alteración de la córnea, a menudo bilateral, que presenta deformidad $y$ adelgazamiento, progresa lentamente y se desarrolla entre la pubertad y los 30 años (1).

\section{Anatomofisiología corneal}

El globo ocular se encuentra conformado por tres capas, la externa, representada por la conjuntiva, esclerótica y la córnea; la media, por la túnica vascular o úvea, la cual comprende a su vez las coroides, el cristalino, el cuerpo ciliar y el iris. La capa interna la conforma la retina, el epitelio no pigmentado del cuerpo ciliar y el epitelio pigmentado del iris. En la porción anterior el área transparente y blanca corresponde a la conjuntiva, la cual se continúa hacía la porción posterior por la esclerótica (2). La córnea es el lente ubicado en la cara anterior y está constituida por tejido transparente, tiene aproximadamente $12.5 \mathrm{~mm}$ de diámetro. La córnea permite la refracción y transmisión de la luz hacía el interior del órgano. Posee dos superficies, una exterior convexa y una interior cóncava, su espesor es de $1 \mathrm{~mm}$ en la periferia y disminuye aproximadamente a la mitad en el área central. En un corte transversal histológicamente está conformada por cinco capas bien delimitadas: el epitelio anterior, la membrana de Bowman, el estroma, la membrana de Descement y el epitelio posterior o endotelio. El epitelio corneal anterior se define como pluriestratificado pavimentoso no queratinizado y está constituido por cinco o seis capas. Dicho epitelio cuenta con gran número de terminaciones nerviosas libres, lo que da razón de la especial sensibilidad corneal (3).

\section{Etiologías posibles}

Se desconoce su etiología precisa y no se han identificado agentes probados epidemiológicamente que influyan en su progresión. Se han determinado índices y valores numéricos que delimitan con un alto grado de fiabilidad los casos sub-clínicos de otro tipo de patologías como astigmatismos irregulares, o las alteraciones originadas por el uso de lentes de contacto (4). Normalmente en el queratocono se produce un adelgazamiento corneal de predomino central o paracentral inferior, el cual causa un aumento progresivo de la curvatura corneal, con miopía, astigmatismo irregular y protrusión apical cónica (5).

Aunque es raro encontrar queratocono e hipermetropía simultáneamente, si el queratocono es producido después del lasik, la hipermetropía no es un factor potencial, para el desarrollo de la ectasia corneal. En nuestra práctica clínica, confirmamos esta aseveración, pues aunque sí se encuentran casos de córneas queratocónicas en pacientes hipermétropes, realmente la incidencia no llega al 1\%. El diagnóstico precoz del queratocono es fundamental, ya que de esto va a depender, en gran parte, el manejo, la evolución y la incidencia de la enfermedad $(6,7)$. Se asocia como ya se mencionó, con enfermedades sistémicas: entre ellas la osteogénesis imperfecta y anomalías cromosómicas como el síndrome de Down, en un 38\%, se asocia con pacientes que tienen prolapso de la válvula mitral.

Dentro de las patologías que más guardan relación con el queratocono se encuentran las enfermedades atópicas. Las complicaciones oculares conocidas como 
dermatitis atópica son blefaritis, queratoconjuntivitis, uveítis, cataratas subcapsulares y desprendimiento de retina. Su frecuencia varía entre el $25 \%$ y el $50 \%$. Un estudio reciente, publicado en la revista Acta Dermoveneorológica de noviembre de 2006, determinó la frecuencia y el tipo de complicaciones oftalmológicas en 37 niños y 22 niñas con dermatitis atópica y con edad promedio de 36.2 meses.

Los objetivos secundarios del estudio eran correlacionar la severidad de la dermatitis atópica, las implicaciones faciales, los signos oculares externos y la presencia de complicaciones oculares e identificar los factores de riesgo para las complicaciones oftalmológicas. Este reveló que en dermatitis atópica cuya severidad era moderada según el índice SCORAD, 15 niños (25.4\%) presentaban signos oculares externos, 1 (1.69\%) tenía una catarata nuclear, $11(18.64 \%)$ presentaron conjuntivitis papilofolicular de tipo benigna, $1(1.69 \%)$ paciente con conjuntivitis bacterial purulenta, $1(1.69 \%)$ con blefaritis atópica crónica y $1(1.69 \%)$ con ambliopía.

El mismo estudio para dermatitis atópicas severas, implicaciones faciales y signos oculares externos pareciera no demostrar influencia en la incidencia de implicaciones oculares. Este estudio sugiere que las complicaciones oculares severas son ciertamente raras en niños pequeños con dermatitis atópica moderada (8). En un estudio reciente sobre la asociación del queratocono y la diabetes como un posible factor de riesgo se concluyó que no existe relación directa, pero entre más avanzada esté la diabetes más severa es la presentación del queratocono.

Dentro de los cambios metabólicos descritos se han encontrado alteraciones en la Interleukina-6, la TNFalfa y MMP-9, las cuales son sobre expresadas en las lágrimas de los pacientes con queratocono, indicando que la patogénesis de este puede implicar eventos inflamatorios crónicos (10). La composición de las laminillas de las córneas normales, (Cadena LMALPHA1- ALPHA5, BETA1-BETA3, GAMMA1-
GAMMA2) y de los botones corneales con queratocono, queratopatía bullosa, distrofia de Fuchs con y sin queratopatía bullosa, y cicatrices corneales, después de queratoplastías lamelares profundas, fueron investigadas con inmunohistoquímica. Las membranas basales epiteliales en córneas normales y en córneas enfermas contenían la cadena L-M ALPHA3, ALPHA5, BETA1, BETA3, GAMMA1, GAMMA2.

La morfología de las membranas basales epiteliales, estaba alterada en las diferentes enfermedades arriba mencionadas. Las cicatrices fueron asociadas con membranas basales irregulares y localización ectópica estromal de las diferentes cadenas de laminillas. La membrana de Descemet, contenía la cadena LMALPHA5, BETA1 y GAMMA1 en todos los casos, y adicionalmente la cadena L-MBETHA3 y GAMMA2 en la mayoría de córneas queratocónicas. La interfase en las córneas con queratoplástia lamelar profunda tenía una cadena LMALPHA3, ALPHA4, ALPHA5, BETA1 Y BETA2 y una estructura extra como la membrana basal, debajo de la membrana de Bowman. Estos resultados sugieren que las cadenas de laminillas participan en el proceso de cicatrización corneal y en la patogénesis de algunas enfermedades corneales. Resultados recientes encontraron la cadena LMALPHA3, BETA3 y GAMMA2 en la Membrana de Descemet, en los botones con queratocono, indicando que esta membrana está involucrada en la enfermedad y que algunos casos de queratocono pueden tener un origen congénito sin una normal hipo regulación de la cadena L-M BETA3 (11)

Las córneas queratocónicas tienen elevados niveles de catepsinas V/L2,-B y -G, que pueden estimular la producción de peróxido de hidrógeno, sobre regulando la catálisis y la enzima antioxidante. Al estar disminuida la enzima TIMP-1 e incrementados los niveles de catepsinas $\mathrm{V} / \mathrm{L} 2$, puede jugar un rol en la degradación de la matriz, que es un sello de las córneas queratocónicas. Los resultados apoyan la hipótesis de que las córneas con queratocono sufren de stress 
oxidativo y degradación de los tejidos (12).

En otro estudio realizado en la Universidad de Arizona en Tucson, sus autores pudieron demostrar por primera vez la expresión de 14.3.3sigma, o stratifin o queratinocito o queratocito específico, un marcador diferencial de células epiteliales, en epitelios corneales humanos. Sin embargo no encontraron diferencias significativas entre los niveles del 14.3.3sigma en córneas sanas, distrofias corneales del queratocono, o pacientes con edema corneal. La immunofluorescencia de 14.3.3sigma fue observada en el citoplasma y en el núcleo de células epiteliales y co-localizado con cyclin-B1 (13). Este trabajo presenta la evidencia de la expresión 14.3.3sigma en epitelio corneal y permite elucidar sobre sus implicaciones posibles en condiciones patológicas corneales (14).

Algunos autores han sugerido como etiología: la baja rigidez escleral, condiciones alérgicas como conjuntivitis vernal o atípica y anormalidades bioquímicas que incluyen disminución de los niveles de glucosa 6 fosfato deshidrogenasa, lo cual disminuye la hidroxilación de la lisina y la glicosilación de la hidroxilisina, disminuyendo el total de colágeno, pero no por esto se puede definir como una enfermedad bioquímica específica (15).

También se dice que es una patología de orden degenerativa normalmente bilateral no inflamatoria de los queratocitos que conlleva a un adelgazamiento y encurvamiento corneal anterior y posterior secundario a una alteración genética autosómica dominante, con penetrancia completa, y expresión altamente variable. De igual modo, se tienen las expresiones disminuidas que se manifiestan como sospecha de queratocono (16)

Los pacientes con queratocono han sido encontrados con fibras nerviosas adelgazadas en el estroma y reducción en la densidad nerviosa en el plexo subepitelial e hipoestesia corneal comparados con pacientes de control (17). A pesar de las alteraciones morfológicas y bioquímicas del queratocono, su etiología sigue siendo confusa. Mientras que los cambios primarios se encuentran principalmente dentro del tejido estromal corneal, algunos autores creen que el epitelio corneal desempeña el papel principal en la formación del queratocono. Por lo tanto, el estudio de la membrana basal epitelial se ha convertido en un aspecto de interés en la investigación del queratocono, encontrar un adelgazamiento basal epitelial masivo en el queratocono es raro, es más probable encontrar corneas guttatas inversas (18).

Así mismo, se identificaron un número de características celulares del queratocono tales como incursiones discretas de procesos celulares finos de los queratocitos anteriores en asociación con la indentación localizada del epitelio basal, y un incremento en los niveles de la enzima lisosomal, catepsina B y $G$ en los queratocitos aberrados, situados debajo de regiones comprometidas de la capa de Bowman y del estroma profundo. La actividad enzimática de estas células parecía causar la degradación estructural localizada del estroma anterior, conduciendo a la destrucción completa de la capa de Bowman y del estroma, haciendo necesario a menudo un injerto corneal para su restauración. Los resultados iniciales de este estudio amplían la investigación del papel de los nervios corneales que pasan entre el estroma y el epitelio a los sitios donde se realizan cambios degradativos iniciales observados previamente, y puede facilitar las interacciones de queratocitos epiteliales en esta enfermedad (19).

Las células en las disecciones de córneas humanas normales y queratocónicas fueron etiquetadas con un fijador fluorescente con tinte de visibilidad 5, el diacetato clorometilfluoresceina, los anticuerpos y la alfa-tubulin (nervios), el integrin alpha3beta1, la catepsina B y G, y el tinte nuclear DAPI, y fueron examinados con un microscopio confocal. Los núcleos de los queratocitos anteriores estaban enrollados alrededor de los nervios, los cuales pasaron a la capa de Bowman a través de otra forma acelular, y mientras que la enfermedad progresaba y la capa de Bowman era degradada, estos queratocitos fueron observados al expresar niveles más altos de catepsina B y G, y luego desplazarse 
anteriormente dentro del epitelio. La localización de los nervios adelgazados dentro del epitelio en asociación con la expresión de catepsina B y G, parecen ser muy destructivos a la córnea. La penetración en los mecanismos moleculares de la patogénesis de la enfermedad del queratocono y su progresión pueden ser obtenidas desde los procesos de la remodelación de la matriz extracelular. Otros estudios son requeridos para determinar los modelos de las bases moleculares en la patogénesis del queratocono (19).

En un estudio sobre la fluorescencia multifotónica y generación de imágenes armónicas secundarias, de las alteraciones estructurales del queratocono en cadáveres, se encontró que las células epiteliales fluorescentes alrededor del área apical estaban elongadas y estaban alineadas paralelamente a las fibras adyacentes del colágeno. La distribución de los patrones paralelos y centrípetos de los paquetes del colágeno estromal, fueron demostrados en diversas profundidades dentro de las córneas queratocónicas. La microscopia multifotónica y el estudio de las imágenes armónicas secundarias proporcionan el análisis estructural tridimensional del queratocono en córneas de cadáver. Esto puede proporcionar importante información morfológica para la investigación de la patogénesis del queratocono y puede ser útil en los hallazgos clínicos, como sistema de diagnóstico y de supervisión in vivo para el avance del queratocono (20).

La proyección de imagen de señales armónicas secundarias microscópicas, combinadas con la reconstrucción tridimensional de la córnea normal, identificó un alto grado de entretejido laminar, particularmente en la córnea anterior. Cabe anotar la importancia en la detección de las laminillas que se insertaron en la capa de Bowman y que estaban orientadas transversalmente a la superficie corneal, penetrando posteriormente a 120 micras. En córneas queratocónicas, la proyección de imágenes de señales armónicas secundarias identificaron menos entretejido laminar y una marcada reducción o una pérdida de laminillas que se insertaban en la capa de Bowman, particularmente en regiones asociadas con el desarrollo del cono sin roturas en la capa de Bowman o cicatrices. Comparando las córneas normales del adulto, las anormalidades marcadas fueron detectadas en la organización de las laminillas del colágeno anterior corneal de las córneas queratocónicas. Estas anormalidades estructurales son constantes con los cambios conocidos en la organización del colágeno y la fuerza biomecánica del queratocono (21).

En estudio realizado por Whitelock y colaboradores del Departamento de Oftalmología y Ciencias Visuales de la Universidad de Illinois y del Chicago College of Medicine, se encontró elevada la actividad de la enzima lisosomal y reducidos los niveles del inhibidor de la alpha1, proteinasa del RNA mensajero, indicando alteraciones metabólicas en pacientes con queratocono (22). Es relevante que en condiciones de queratocono, significativamente se encontró mayor apoptosis en las células del estroma anterior de córneas con queratocono, y en la mayoría TIMP-1 y TIMP-3, producidas en las células estromales, localizadas en la misma región en relación con las córneas normales (23).

En un estudio realizado por el Departamento de Oftalmología, Aarhus University Hospital, Aarhus, en Dinamarca se encontró que algunos genes pueden estar implicados en la formación del queratocono. Dos de los genes candidatos, el lisil oxidasa y el inhibidor del tejido fino de la metalloproteinasa 3, se sabe están implicados en otras enfermedades del ojo. Los perfiles de expresión fueron reproducidos con el software dChip (laboratorio de Wong) y PCR en tiempo real. Los aumentos en la queratina 6 y 13 también fueron detectados en los niveles de proteína. El epitelio de la córnea queratocónica apareció con cambios masivos del citoesqueleto, reducción de la matriz extracelular remodelada, signos de alteración de la transmembrana e interacciones modificadas de célula-a-célula y de célula-a-matriz. La validación de la expresión del gen con análisis del dChip y PCR en tiempo real indica que 
el GenChip ha de ser validado con la técnica para la investigación del epitelio con muestras de córneas disecadas. La asociación entre las alteraciones en el RNA y los niveles de proteína fue observada para algunos de los candidatos examinados (24).

El queratocono y la catarata son causas comunes de la morbilidad visual. Ambas condiciones demuestran la predisposición genética. El locus para el queratocono con la catarata fue mapeado en la región $6.5-\mathrm{Mb}$ del brazo largo del cromosoma 15, en 22.33-24.2 entre CYP11A y D15S211. Los genes candidatos posicionales y funcionales CTSH, CRABP1, IREB2, y RASGRF1 fueron excluidos como la causa del queratocono con la catarata en esta familia. Éste es el primer informe de una familia con la herencia dominante autosomal del queratocono en asociación con catarata. Los mapas causantes del gen del brazo largo del cromosoma 15, aún no se han identificado (25). Otro estudio nos habla de la ubicación del gen en pacientes con queratocono, localizado en la región cromosomal 16q22.3-q23.1(26).

La causa del queratocono es incierta. Un reporte inicial demostró una deficiencia del magnesio en pacientes con queratocono, y sugirió que la insuficiencia de este elemento puede afectar patológicamente el mecanismo de la córnea. Documentos experimentales y clínicos concernientes a la posible relación entre queratocono y la deficiencia de magnesio fueron revisados. Estos estudios demuestran una alteración celular y molecular específicas de la córnea queratoconica, incluyendo un adelgazamiento y fragmentación de las membranas, degeneración celular y de las fibras de colágeno, cambios mitocondriales y una anormalidad bioquímica en la síntesis de proteínas (27). En Argentina al queratocono se le conoce como Síndrome de Thalasselis, por ser esta investigadora quien definió la relación entre la deficiencia de Zinc y los pacientes con queratocono.

Lipman y colaboradores concluyeron que el queratocono y la distrofia corneal de Fuchs pueden ocurrir en un mismo paciente, dentro de sus observaciones consideraron que el queratocono puede ser hereditario y que la distrofia de Fuchs es más frecuente en el género femenino con un patrón de herencia autosómica dominante (28) pero hasta el momento no se han puesto de acuerdo los diferentes autores en cuanto al género en el cual predomina la patología, algunos afirman que en informes estadísticos la patología se presenta más frecuentemente en mujeres que en hombres mientras que para otros autores la patología es más frecuente en el género masculino (29).

El concepto de que existen cambios estructurales en el estroma corneal, producidos por el uso de los lentes de contacto, es soportado por varios estudios, los cuales encuentran correlación entre la reducción del espesor, un incremento en la protrusión y gran irregularidad a nivel corneal en los usuarios de lentes de contacto (29). Dentro del trabajo que realiza el grupo Investigadores en Optometría de la Universidad Antonio Nariño se plantea como posible factor de riesgo, el moldeado de la córnea por el uso de lentes de contacto lo cual puede llegar a ocasionar queratocono, esto evidenciado por los pacientes atendidos por el grupo.

Gráficos seriados de los queratoconos progresivos y de los moldeados corneales regresivos inducidos por los lentes de contacto, demostraron que el vector de desplazamiento de los valores del coeficiente de irregularidad corneal (CIC) y el coeficiente de poder corneal (CPC) pueden ser potencialmente usados, principalmente para distinguir, el moldeado corneal inducido por los lentes de contacto, en córneas afectadas por queratocono. El diagrama queratomórfico (topografía) ayuda en la clasificación y la comparación de la forma corneal por los índices de los ejes gráficos y utilizando las escalas conocidas. El diagrama puede ser utilizado como una herramienta para la valoración prequirúrgica de la estabilidad de la superficie corneal y en el postoperatorio como valoración de la progresión y de los cambios en la forma corneal producidos por la cirugía (30).

www.unicolmayor.edu.co 


\section{Situación epidemiológica global}

El origen de la enfermedad no se encuentra claramente definido, pero existen varias teorías que intentan explicar su presentación. Mientras en otros países su incidencia es según 50-230x100,000 y la prevalencia 54.5x100,000 (31), en Colombia no se cuenta con estudios epidemiológicos que den cuenta de la verdadera dimensión del problema. Sin embargo, existe entre los profesionales, oftalmólogos y optómetras, alguna evidencia que señalaría un incremento importante de la frecuencia de casos diagnosticados en consulta.

\section{Factores de riesgo}

Existen factores extrínsecos e intrínsecos que parecen influir en los pacientes portadores de queratocono, es el interés de este grupo de investigación entrar a determinar cuáles y de qué forma intervienen en la aparición del mismo. El examen de agudeza Visual es fundamental pero no es determinante para diagnosticar que ese paciente pueda estar exento de presentar algún tipo anomalía ocular. Aún así, la deficiencia en la agudeza visual constituye la tercera causa de morbilidad en nuestro país, como ya se mencionó (32). Normalmente la disminución de la agudeza visual, acompañada de algunos otros signos oftalmoscópicos, retinoscópicos y queratométricos típicos nos permiten el diagnóstico de queratocono. Entre las conclusiones del estudio de Avendaño y Rodríguez encontró que la agudeza visual está asociada con la edad, el sexo, y es independiente del ingreso económico y del nivel de urbanización (33).

Entre los Factores de riesgo, están las asociaciones con enfermedades sistémicas: síndrome de Apert, síndrome de Rieger, osteogénesis imperfecta, prolapso de la válvula mitral, síndrome de Crouzon, enfermedades atópicas, enfermedades del colágeno; patologías oculares: amaurosis congénita de Leber, aniridia, síndrome de Marfán, retinosis pigmentaria, síndrome del párpado colgante; anomalías cromosómicas: síndrome de Down; alteraciones metabólicas: síndrome de Thalasselis o deficiencia al zinc asociada con personalidad tipo A y aumento en las catepsinas.(33).
Es indispensable llegar a conocer las relaciones entre la salud visual y ocular y factores tales como los hábitos higiénicos, el ambiente, las condiciones de trabajo, la iluminación, la alimentación, la disponibilidad de servicios de salud, los hábitos de lectura, etc., a fin de complementar el diagnóstico del problema, aplicar la tecnología disponible en Colombia, y diseñar apropiadas alternativas de solución, de máxima cobertura y costos razonables, incluyendo la capacitación de personal de nivel profesional, generando un impacto social que permita establecer políticas de Salud (33)

\section{Perspectivas de investigación}

Los optómetras y oftalmólogos que atienden pacientes con queratocono hacen énfasis en el cuidado médico y óptico. En un estudio realizado por I. Wahrendorf sobre como vive un paciente con queratocono, las conclusiones revelaron que la relación entre especialista y paciente es fundamental. Estos pacientes manejan una relación visual entre deficiencia, minusvalía visual y normalidad. El tratamiento más importante de compensación para los pacientes con queratocono actualmente es el lente de contacto rígido gas permeable. Con su ayuda, la mayor parte de pacientes disfruta de una visión normal, que produce un efecto favorable sobre todos los aspectos de la vida. Si el queratocono se desarrolla en forma agresiva las restricciones en calidad de la vida son más severas que en el caso de un desarrollo lento de la enfermedad. Contrariamente a la hipótesis propuesta en la investigación de Wahrendorf la existencia de una red social estrecha en torno al paciente no tiene un efecto de alivio significativo sobre las restricciones visuales sentidas. Una comunicación comprensiva del diagnóstico, sin embargo, tendrá un efecto favorable sobre el paciente en la aceptación de la condición y la integración del queratocono en su vida (34).

\section{Visión particular en el caso colombiano}

Sobre la salud visual de los colombianos se han hecho algunos estudios importantes a nivel regional en cuanto 
a diagnóstico; lamentablemente no existe una base de datos que los agrupe a todos; el único estudio de magnitud nacional data de hace ya dos décadas y tiene datos útiles, pero no cubre el asunto que aquí se trata (35).

\section{Métodos de diagnóstico}

Es importante tener en cuenta que para el diagnóstico del queratocono es necesario realizar un estudio de la topografía corneal del paciente. Los parámetros de elevación de cara anterior, cara posterior, paquimetría y profundidad de cámara anterior, medidos en la topografía de elevación, difieren significativamente entre individuos normales y aquellos con queratocono clínico y sub-clínico. Estos parámetros pueden ser de utilidad para definir pacientes con riesgo de desarrollar una ectasia secundaria a cirugía refractiva corneal. Con más exactitud se utiliza la topografía corneal, en casos incipientes. La topografía típica, muestra un marcado aplanamiento de la cornea, a través del eje vertical y en la periferia inferior sitio de la lesión. Un estudio que utilizó la topografía en familias genéticamente establecidas con queratocono permitió que se detectaran los patrones topográficos sospechosos lo que trae nuevos datos a la tarea difícil de diagnosticar queratocono frustro (36).

Las distrofias y degeneraciones corneales incluyen, la megalocórnea, el queratoglobo, el queratotoro o pseudo queratocono pelúcido (37), la degeneración marginal pelúcida y sus combinaciones. En la megalocórnea la córnea es mucho más grande en diámetro y su espesor es normal. El queratoglobo presenta un adelgazamiento del limbo al limbo. El queratotoro se caracteriza por una disminución del espesor corneal periférico en $360^{\circ}$ (38). La degeneración marginal pelúcida es un desorden no inflamatorio, progresivo, caracterizado por una banda delgada periférica localizada en la córnea inferior limbar, acompañada de uno o dos milímetros de banda corneal normal.

El Pentacam (Oculus) y el uso de nuevas tecnologías, han hecho más fácil el diagnóstico de la ectasia. Este equipo compensa la asfericidad y en los mapas topográficos y tomográficos, permiten diagnosticar tempranamente, el queratocono. Los procedimientos de topografía y tomografía son mejores para detectar el adelgazamiento corneal en casos incipientes de queratocono y degeneración marginal pelúcida, ya que detectan las diferencias de espesores con gran precisión (39).

La Universidad de Illinois en su departamento de Oftalmología y Ciencias Visuales, realizó un estudio para determinar un nuevo método para medir la severidad del queratocono. Un sistema que calificaba simple y confiable para el Queratocono y que podía ser útil en los estudios genéticos para un rasgo complejo que requería una medida cuantitativa de presencia o similitud y de severidad de la enfermedad. El método desarrollado o KSS utilizó 2 índices corneales de la topografía: el poder corneal promedio y el error corneal medio de las aberraciones de alto orden (RMS) de los polinomios de Zernike derivados del primer frente de onda superficial corneal. Las muestras clínicas incluyeron las estrías de Vogt, los anillos de Fleischer y las cicatrices corneales (40). Cada día hay más elementos de diagnóstico, hay mayor esclarecimiento y conocimiento de las complicaciones oculares y los signos clínicos, desafortunadamente no es así en la determinación de la etiopatogenia del cono.

La progresión paquimétrica constituye un punto de evaluación tradicional del espesor corneal y debe ser considerada como prueba tamiz para los candidatos a cirugía refractiva. El encontrar valores paquimétricos inferiores o superiores a los valores normales que oscilan entre 490 y 560 nanómetros constituye un punto de alerta o alarma entre los profesionales de la salud visual y ocular. Existe una gran variabilidad en los valores paquimétricos en pacientes con queratocono. Este parámetro representa un índice indirecto de la biomecánica del tejido corneal, pero la hipótesis de que las córneas queratocónicas sufren una mayor progresión de los valores paquimétricos comparadas con córneas normales requiere mayores estudios (41).

Investigaciones recientes utilizaron la medición de las aberraciones de alto orden, a través del frente de onda; en un estudio prospectivo comparativo de casos y 
controles, realizado en un hospital universitario, utilizando una muestra de 23 ojos con queratocono incipiente, formando el grupo I, y 10 ojos asintomáticos y sin indicio de queratocono pero con topografías anormales, formando el grupo II, 127 ojos sanos de 74 pacientes con signos negativos, que conformaron el grupo de control, grupo III. A todos se les practicó una descomposición de séptimo orden de los polinomios de Zernike, para determinar la aberración de la primera superficie corneal. El coeficiente sencillo de las aberraciones de alto orden de la prueba de Zernike arrojó como resultado, el índice Z-3, y con el uso del análisis discriminante D-13, para los grupos 1 y $3, \mathrm{D}(23)$, grupos 2 y 3 , determinaron la discriminación existente entre los ojos normales clínicamente y los ojos con queratocono. Clínicamente el seguimiento en ojos normales y ojos con queratocono incipiente, demuestran diferencias significativas, en las aberraciones de la primera superficie, comparadas, entre ojos normales y aquellos que posteriormente puedan ser considerados como ojos con queratocono sub-clínico (42).

\section{Tratamiento}

El queratocono es una de las queratopatías más frecuentes, en la práctica clínica es recomendable el uso de lentes de contacto de tipo rígidos y cuando no son tolerados, se debe buscar otras alternativas en el tratamiento. Según estudios recientes, se está utilizando un tipo de lente de contacto denominado ROSE-K. El promedio simulado de la curvatura corneal, en el mapa de la topografía axial, predice la curva base final de la adaptación del lente de contacto significativamente mejor que con la topografía tradicional. Los lentes de contacto ROSE-K rígidos fueron adaptados con éxito en el $100 \%$ de los casos de queratocono moderado y en el 96\% de los queratoconos severos. El 90\% de los casos reportaron comfort y la determinación de la curvatura axial corneal, por medio de la topografía es mucho mejor para determinar la curva base del lente final (43).

El Rose-K es un lente rígido gas permeable, escleral, pentacurvo, diseñado por el Dr. Rose, de curva base
8.7, que en la actualidad puede ser comparado con el Sclerakon oscicon- 150 o con el Soft-perm o lente saturno (44). Se han descrito diferentes casos de adaptación de lentes de contacto esféricas, de alta excentricidad, para adaptaciones de queratocono $(45,46)$. Dentro de nuestra práctica clínica hasta el momento contamos con gran variedad de diseños de lentes de contacto rígidos gas permeables. Para lograr buenas adaptaciones en córneas queratocónicas, es necesario entonces tener en cuenta las necesidades del paciente.

Una nueva alternativa en el tratamiento del queratocono y para lograr mayor estabilidad corneal, es el CrossLinking o fotoablación corneal del colágeno por radiación ultra violeta tipo A (UVA) más riboflavina. Para evitar daño de la irradiación de UVA al endotelio corneal, los parámetros para el procedimiento se fijan de una manera que el tratamiento eficaz ocurre solamente en las primeras 300 micras del tejido estromal corneal. Esta fotoablación no sólo consolida las características biomecánicas de la córnea sino que también induce apoptosis de los queratocitos. Hasta la fecha, la eficacia del tratamiento se puede supervisar solamente con los seguimientos indirectos por las topografías corneales post-operatorias o usando microscopia confocal corneal (46). Estos estudios recientes han demostrado una línea específica de demarcación que se observa entre el tejido tratado y el no tratado con la valoración por lámpara de Hendidura y permite observar las diferencias en los puentes de unión.

En la Investigación cualitativa, el tratamiento conservador del queratocono a través de cross-linking inductor del colágeno con UVA más Riboflavina con el microscopio confocal HRT II, fue confirmada la restauración del epitelio y la re-inervación posterior al tratamiento directamente en vivo en tejido corneal humano en pacientes con queratocono. El tratamiento incluyó la instilación de $0.1 \%$ de riboflavina en $20 \%$ de solución de dextrosa, 5 minutos antes de la irradiación de UVA y cada 5 minutos para un total de 30 minutos. La energía radiante era $3 \mathrm{~mW} / \mathrm{cm} 2$ o 5.4 julios $/ \mathrm{cm} 2$ y la fuente eran UVA dual (370 nanómetros) LED 
luminescente (47). Se le considera uno de los últimos avances en el tratamiento del cono corneal, sin embargo solo a largo plazo se podrá medir los efectos secundarios de la irradiación corneal, la aparición de pterigios, cataratas u otros.

El Cross Linking con colágeno y UVA más Riboflavina, seguidos de una ablación utilizando el Femtosecond Láser, demostró una segura y efectiva técnica para la rehabilitación visual en ojos con queratocono o ectasia postlasik. El propósito de la ablación no es lograr la emetropia, es tratar de normalizar la córnea, la topografía guiará el tratamiento para aplanar el ápice corneal, de igual manera hacerlo extensivo hasta la periferia. Para un mejor resultado se deben utilizar los nomogramas de ajuste en cuyos casos el tratamiento es menor de lo planeado. Con relación a las nébulas presentadas en unos pocos ojos, un estudio revela que se deben a problemas de cito toxicidad en el tratamiento con UVA, y el Cross Linking, este procedimiento no debe ser utilizado en ojos con espesor corneal, inferior a 400 nanómetros, y que presente daños en las células endoteliales (48).

Otra forma de tratamiento donde se pretende estabilizar el tejido corneal consiste en la inserción de anillos intraestromales en la córnea, los cuales según estudios, han dado muy buenos resultados en los pacientes sometidos a esta técnica. En un trabajo de investigación clínica los anillos intraestromales fueron implantados en ojos de pacientes con queratocono sin complicaciones intraoperatorias. Los anillos proporcionaron una mejoría en la miopía y un astigmatismo regular en los pacientes con queratocono. Dentro de los resultados se encontró un queratocono más estable, un astigmatismo $<3.50 \mathrm{D}$ y una miopía esférica significativa $(>-1.75)$ lo que predicen un mejor resultado (49). Sin embargo, la recomendación ideal para este tipo de cirugía es en casos de queratoconos leves o moderados.

El buen resultado visual de pacientes con queratocono fue logrado cuando se utilizó un retoque con el sistema Femtosecond Láser, creando canales para la implantación de anillos intraestromales corneales. El retoque con Femtosecond láser produce unos efectos muy grandes en la queratometría y en el equivalente esférico. Hay una mejoría en la agudeza visual, seis meses después de la implantación comparando con el grupo mecánico (sin Femtosecond Láser), en el cual al comienzo presentó buena agudeza visual, pero más tarde esta agudeza visual decayó (50).

El tratamiento quirúrgico debe ser contemplado como la última alternativa que se ofrece a un paciente con queratocono. La existencia de queratocono en pacientes intervenidos con queratoplastía penetrante y que no tenían antecedentes ni preexistencia de queratocono, en dos casos, sugieren la transmisión del desorden desde la córnea del donante (51).

Pacientes con córneas queratocónicas, a los cuales se les implantaron injertos corneales longitudinales (lamelares) presentaron edema corneal, y fueron examinados cuidadosamente, no solo por sus signos del rechazo al injerto, sino por los agudos hidrops que presentaron. Importante diferenciar estos signos causados por el rompimiento o desprendimiento de la Membrana de Descemet y la presencia de edema estromal, a través del tejido del donante (52).

El factor de riesgo más importante para un rechazo después de un transplante corneal por queratocono, fue el tamaño del injerto, los investigadores concluyeron que afortunadamente la progresión para el rechazo puede ser prevenible, si el tratamiento se inicia intensiva y tempranamente (53). Los lentes de contacto blandos, en la práctica clínica, revelan una mejor tolerancia que los rígidos gas permeables en su adaptación, pero desafortunadamente los estudios realizados muestran las evidencias de no satisfacción de las necesidades de agudeza visual necesarias de los pacientes.

Es importante tener en cuenta que existen diferentes técnicas en el tratamiento del queratocono, las cuales deben ser evaluadas con sumo cuidado, para lograr estabilidad corneal y si es posible una mejoría en la agudeza visual, además de atender las necesidades de los pacientes y seleccionar de acuerdo al caso clínico 
cual es la mejor alternativa en el tratamiento del mismo. Como grupo de investigación seguiremos en la búsqueda permanente de nuevos desarrollos científicos y tecnológicos que permitan al paciente y al profesional afrontar este difícil reto.

\section{Síntesis}

1. No existen evidencias que permitan aseverar, la incidencia genética en la presencia de Queratocono.

2. La adecuada adaptación de Lentes de Contacto es fundamental para el control de estadios iniciales de queratocono. Pero una inadecuada adaptación se convierte en un factor de riesgo por el moldeado corneal que induce.

3. Es muy importante utilizar los métodos de diagnóstico que actualmente existen para confirmar el diagnóstico precoz del queratocono e iniciar su manejo y control inmediatamente.

4. Actualmente en Colombia se están utilizando tecnologías de punta para el manejo y control del queratocono como por ejemplo, los Lentes de Contacto de diseño especial, los Anillos Intaestromales, el Cross Linking y el tratamiento con el Femtosegundo.

5. En Colombia el incremento en la prevalencia e incidencia de esta afección está llegando a convertirse en un problema de Salud Pública, la Universidad Antonio Nariño y su Facultad de Optometría pretenden con el desarrollo de una investigación sobre los factores de riesgo de esta alteración, establecer sus etiologías asociadas para presentar políticas de prevención y promoción en la Salud Visual y Ocular de la población colombiana.

6. Determinar la etiología y evidenciar los factores de riesgo desencadenantes se convierte en meta de corto, mediano y largo plazo para todos los investigadores interesados en el tema.

\section{Referencias}

1. Velarde J. Queratocono Subclínico en paciente portador de lentes de contacto, candidato a cirugía refractiva, 20 meses de seguimiento topográfico. Rev Española de contactología. 2000;10: http://www.oftalmo.com/sec/00-tomo-1/08.htm.

2. Cadena D. Manual de Anatomia Humana. Tercera Edición, Celsus 1992, p 344-345.

3. Arffa R. Enfermedad de la Córnea. 4ta ed. España: Brace Publishers International. 1999; p1-19.

4. Lebow KA, Grohe RM. Differentiating contact lens induced war page from true keratoconus using corneal topography. CLAO J 1999;25:114-122.

5. Barraquer R, De Toledo M, Torres E. Distrofias y degeneraciones corneales. Barcelona: España; 2004; p13.

6. Abad J, Awad A, Kurstin J. Hyperopic keratoconus. J Refract Surg. 2007;23:520-523

7. Díaz G, Caíñas A, Jiménez C, Neira P. Características epidemiológicas en pacientes portadores de queratocono Rev Cubana Oftalmol 1999;12:20-26.

8. Carmi E, Defossez-Tribout C, Ganry O, Cene S, Tramier B, Milazzo S, Lok C.Acta Derm Ocular complications of atopic dermatitis in children. Venereol. 2006;86:515-517.

9. Kuo IC, Broman A, Pirouzmanesh A, Melia M Is there an association between diabetes and keratoconus? Ophthalmology. 2006;113:184-190.

10. Lema I, Durán J. Inflammatory molecules in the tears of patients with keratoconus. Ophthalmology. 2005;112:654-659.

11. Byström B, Virtanen I, Rousselle P, Miyazaki K, Lindén C, Pedrosa Domellöf F. Laminins in normal, keratoconus, bullous keratopathy and scarred human corneas Histochem Cell Biol. 2007; 127:657-667.

12. Kenney MC, Chwa M, Atilano SR, Tran A, Carballo M, Saghizadeh M, Vasiliou V, Adachi W, Brown DJ Increased levels of catalase and cathepsin V/L2 but decreased TIMP-1 in keratoconus corneas: evidence that oxidative stress plays a role in this disorder Invest Ophthalmol Vis Sci. 2005;46:823-832.

13. Dellambra E, Patrone M, Sparatore B, Negri A, Ceciliani F, Bondanza S, Molina F, Cancedda FD, De Luca M. Stratifin, a keratinocyte specific 14-3-3 protein, harbors a pleckstrin homology $(\mathrm{PH})$ domain and enhances protein kinase $\mathrm{C}$ activity. J Cell Sci. 1995;108:3569-3579.

14. Zanello SB, Nayak R, Zanello LP, Farthing-Nayak P. Identification and distribution of 14.3.3sigma (stratifin) in the human cornea. Curr Eye Res. 2006;10:825-833.

15. Rosas E. Queratocono. Franja Visual. 1991;3:15-17.

16. De La Torre A. PRK y LASEK en sospechosos de queratocono. Colom Med. 2004;35:46-49.

17. Simo Mannion L, Tromans C, O’Donnell C. An evaluation of corneal nerve morphology and function in moderate keratoconus Cont Lens Anterior Eye. 2005 ;28:185-192.

18. Rohrbach JM, Szurman P, El-Wardani M, Grüb M. About the frequency of excessive epithelial basement membrane thickening in keratoconusKlin Monatsbl Augenheilkd. 2006;223:889-893. 
19. Brookes N, Loh I, Clover G, Poole C, Sherwin T. Involvement of corneal nerves in the progression of keratoconus. Exp Eye Res.2003;77:515-24.

20. Teng SW, Tan HY, Peng JL, Lin HH, Kim KH, Lo W, Sun Y, Lin WC, Lin SJ, Jee SH, So PT, Dong CY. Multiphoton autofluorescence and second-harmonic generation imaging of the ex vivo porcine eye. Invest Ophthalmol Vis Sci. 2006;47:1216-1224.

21. Morishige N, Wahlert AJ, Kenney MC, Brown DJ, Kawamoto K, Chikama T, Nishida T, Jester JV. Second-harmonic imaging microscopy of normal human and keratoconus cornea. Invest Ophthalmol Vis Sci. 2007;48:1087-1094.

22. Whitelock RB, Fukuchi T, Zhou L, Twining SS, Sugar J, Feder RS, Yue BY. Cathepsin G, acid phosphatase, and alpha 1proteinase inhibitor messenger RNA levels in keratoconus corneas. Invest Ophthalmol Vis Sci. 1997;38:529-534.

23. Matthews FJ, Cook SD, Majid MA, Dick AD, Smith VA Changes in the balance of the tissue inhibitor of matrix metalloproteinases (TIMPs)-1 and -3 may promote keratocyte apoptosis in keratoconus. Exp Eye Res. 2007;84:1125-1134.

24. Nielsen K, Birkenkamp-Demtröder K, Ehlers N, Orntoft TF. Identification of differentially expressed genes in keratoconus epithelium analyzed on microarrays. Invest Ophthalmol Vis Sci. 2003;44:2466-2476.

25. Hughes AE, Dash DP, Jackson AJ, Frazer DG, Silvestri G. Familial keratoconus with cataract: linkage to the long arm of chromosome 15 and exclusion of candidate genes. Invest Ophthalmol Vis Sci. 200;44:5063-5066.

26. Tyynismaa H, Sistonen P, Tuupanen S, Tervo T, Dammert A, Latvala T, Alitalo T. A locus for autosomal dominant keratoconus: linkage to 16q22.3-q23.1 in Finnish families. Invest Ophthalmol Vis Sci. 2002;43:3160-3164.

27. Thalasselis A. The possible relationship between keratoconus and magnesium deficiency. Ophthalmic Physiol Opt. 2005;25:7-12.

28. Lipman RM, Rubenstein JB, Torczynski E. Keratoconus and Fuchs' endothelial dystrophy. Cornea. 1991;10:368.

29. Bergmanson JP, Sheldon TM, Goosey JD. Fuchs' endothelial dystrophy: a fresh look at an aging disease. Ophthalmic Physiol Opt. 1999;19:210-222.

30. Ventocilla M, Stamler J. Contact Lens Complications. www.emedicine.com. 2006.

31. Maeda N, Klyce SD, Smolek MK, Thompson HW. Automated keratoconus screening with corneal topography analysis. Invest Ophthalmol Vis Sci. 1994;35:2749-2757.

32. Rabinowitz YS. Intacs for keratoconus. Curr Opin Ophthalmol. 2007;18:279-283.

33. Avendaño J, Rodríguez E. «La Agudeza Visual en Colombia ENS Instituto Nacional de Salud. 1981.

34. Wahrendorf I. How to live with keratoconus. Klin Monatsbl Augenheilkd. 2006;223:877-888.

35. Yepez A. Salud visual en población menor de 15 años. Programa de atención primaria en Salud Antioquia, Colombia. Ia Treia. 1989; 2:201-206.
36. Arntz A, Durán JA, Pijoán JI. Subclinical keratoconus diagnosis by elevation topography Arch Soc Esp Oftalmol. 2003;78:659-664.

37. Levy D, Hutchings H, Rouland JF, Guell J, Burillon C, Arné JL, Colin J, Laroche L, Montard M, Delbosc B, Aptel I, Ginisty H, Grandjean H, Malecaze F. Videokeratographic anomalies in familial keratoconus. Ophthalmology. 2004;111:867-874.

38. Abad JC, Rubinfeld RS, Del Valle M, Belin MW, Kurstin JM Vertical D: a novel topographic pattern in some keratoconus suspects. Ophthalmology. 2007;114:1020-1026.

39. Rosas E. Médico cirujano Oftalmólogo. Franja Visual, 1991;3:15-17.

40. Charter L. Ophthalmology Times, Cleveland,2006;31:51-52.

41. McMahon TT, Szczotka-Flynn L, Barr JT, Anderson RJ, Slaughter ME, Lass JH, Iyengar SK; CLEK Study Group. A new method for grading the severity of keratoconus: the Keratoconus Severity Score (KSS).Cornea. 2006;25:794-800.

42. Luz A, Ursulio M, Castañeda D, Ambrósio R Jr. Corneal thickness progression from the thinnest point to the limbus: study based on a normal and a keratoconus population to create reference values.Arq Bras Oftalmol. 2006;69:579-583.

43. Bühren J, Kühne C, Kohnen T. Defining subclinical keratoconus using corneal first-surface higher-order aberrations. Am J Ophthalmol. 2007;143:381-389.

44. Jain AK, Sukhija J. Rose-K contact lens for keratoconus Indian J Ophthalmol. 2007;55:121-125.

45. Hernández P. Reinventando las lentes semiesclerales. Gaceta Óptica,2006;402:20-25.

46. González-Pérez J, Arrojo N, Parafita MA. Adaptación de LC asférica de alta excentricidad modificada en queratocono unilateral. Rev. Esp. Contact. 2005;12:69-73.

47. Seiler T, Hafezi F. Corneal cross-linking-induced stromal demarcation line Cornea. 2006;25:1057-1059.

48. Mazzotta C, Traversi C, Baiocchi S, Sergio P, Caporossi T, Caporossi A. Conservative treatment of keratoconus by riboflavin-uva-induced cross-linking of corneal collagen: qualitative investigation. Eur J Ophthalmol. 2006;16:530-535.

49. Guttman Ch. Ophthalmology Times, Cleveland, vol 32 ISS 7 pag 40-41 (2pp). 2007.

50. Levinger S, Pokroy R. Keratoconus managed with intacs: oneyear results. Arch Ophthalmol. 2005;123:1308-1314.

51. Charter, Lynda. Ophthalmology Times, Cleveland, vol 32 ISS 4 pag 42-43 (2pp).2007.

52. Unal M, Yücel I, Akar Y, Akkoyunlu G, Ustünel I. Recurrence of keratoconus in two corneal grafts after penetrating keratoplasty Cornea. 2007;26:362-364.

53. Ezra DG, Mehta JS, Allan BD. Late corneal hydrops after penetrating keratoplasty for keratoconus. Cornea. 2007;26(5):639-640.

54. Científicos del Colegio Médico Jeferson, Universidad Thomas Jefferson. Health and Medicine Week. Atlanta, pag 243. 2007.

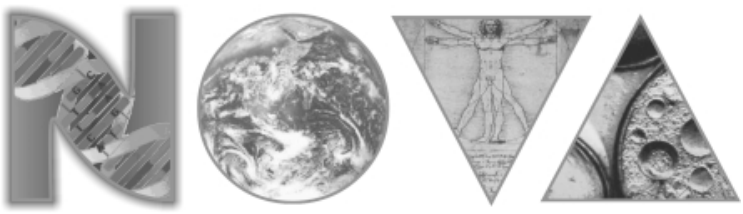

